\title{
RETROPERITONEAL SCHWANNOMA: CASE SERIES AND LITERATURE REVIEW
}

José Cury, Rafael Ferreira Coelho, Miguel Srougi

\section{INTRODUCTION}

Schwannomas are usually benign tumors arising from Schwann cells of the peripheral nerve sheath. ${ }^{1}$ Most schwannomas are benign, although malignant cases are known to occur, especially when there is association with Von Recklinghausen's disease, which occurs in $5 \%$ to $18 \%$ of cases. ${ }^{2}$ In the absence of von Recklinghausen's disease, these masses rarely occur in the retroperitoneum, comprising $0.5 \%$ to $5 \%$ of all schwannomas. ${ }^{3,4}$ Involvement of the urinary tract is rare, but involvement of the testis, penis, spermatic cord, and tunica vaginalis has been previously reported. ${ }^{4}$ We present here our experience with 3 cases of retroperitoneal schwannoma with long-term follow-up and a literature review.

\section{CASE REPORTS}

\section{Case 1}

A 59-year-old white man presented with a long-term history of vague left flank pain. On examination, he had a palpable mass and mild tenderness in left flank. Ultrasonography revealed a $12-\mathrm{cm}$ heterogenous mass in the left adrenal topography, with cystic and necrotic areas. (Figure 1- A). A computed tomography scan confirmed a $10.5 \times 11.5 \times 9.0$ $\mathrm{cm}$ enhancing mass with cystic and necrotic central areas displacing the left kidney inferolaterally (Figure 1- B). The patient was initially diagnosed as having a left adrenal tumor and underwent a mass excision through a left flank incision, between the $11^{\text {th }}$ and $12^{\text {th }}$ ribs. The mass was dissected from the retroperitoneum but was tightly adherent to the left kidney. The left adrenal, left kidney, and the mass were excised en bloc. The postoperative period was uneventful. Macroscopic

Division of Urology, University of Sao Paulo Medical School, Sao Paulo, SP, Brazil.

Email: coelhouro@yahoo.com.br

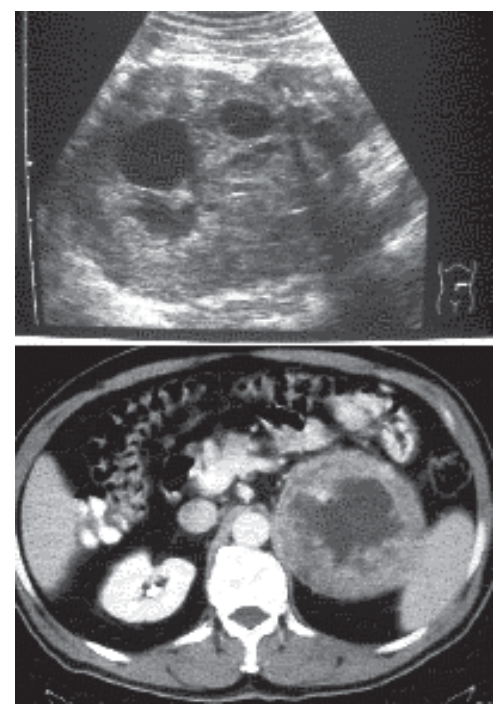

Figure 1-A - Ultrasonography reveals a 12-cm mass on the left flank at adrenal topography with central areas of necrosis and cystic degeneration. B - Computed tomography showing a 12-cm enhancing mass with cystic and necrotic areas arising from the retroperitoneum and displacing the left kidney inferiorly (not shown on this plate) .

examination revealed a capsulated mass of $12 \times 9.0 \times 8.5 \mathrm{~cm}$, with cystic and solid bright-yellow areas. The adrenal was unremarkable, and the left kidney was adhered but not invaded by the retroperitoneal mass. On microscopic examination, the solid mass consisted of a proliferation of fusiform cells that formed a palisade pattern in some regions (Antoni type A). Other regions were composed of mixoid and degenerative tissue with fewer cells and gelatinous substance (Antoni type B) (Figure 2). The cells were diffusely and strongly positive for $\mathrm{S} 100$ protein. The tumor was diagnosed as a schwannoma. At the 12-year follow-up exam, the patient remained asymptomatic, and there was no evidence of recurrence.

\section{Case 2}

A 26-year-old white woman presented with a history 


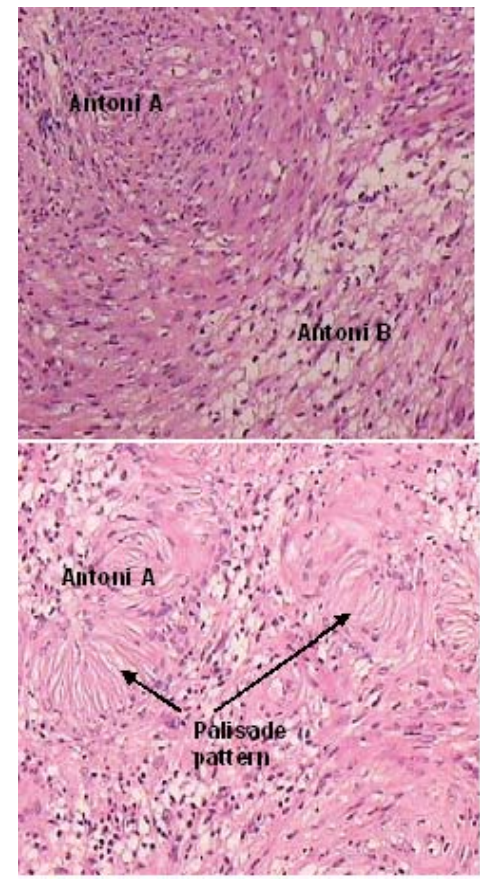

Figure 2 - Microscopic examination. The solid mass consisted of a proliferation of fusiform cells which formed a palisade pattern in some regions (Antoni type A). Other regions were composed of mixoid and degenerative tissue with fewer cells and gelatinous substance (Antoni type B). (HE; 200x)

of vague left flank pain. Physical examination was unremarkable. Ultrasonography revealed a 6-cm heterogeneous expansive mass in left adrenal topography. A computed tomography scan confirmed a $6.5 \times 5.0 \times 4.3-\mathrm{cm}$ enhancing heterogeneous mass with necrotic central areas arising from left adrenal topography. The patient was initially diagnosed as having a left adrenal tumor and underwent a mass excision through a left flank incision including the excision of the $10^{\text {th }}$ rib. The left adrenal, left kidney, and their hilar structures were carefully dissected free from the mass and spared. The capsulated retroperitoneal mass was excised en bloc, without any sign of surrounding tissue invasion. The postoperative period was uneventful. Macroscopic examination revealed a capsulated mass of $7.0 \times 5.5 \times 3.5 \mathrm{~cm}$, with solid bright-yellow areas. Microscopic examination revealed focal areas of Antoni types A and B tissue. The cells were immunoreactive for $\mathrm{S} 100$ protein. The tumor was diagnosed as a schwannoma. At the 12-year follow-up exam, the patient remained asymptomatic, and there was no evidence of recurrence.

\section{Case 3}

A retroperitoneal hypoechoic $3-\mathrm{cm}$ mass was incidentally detected in 68-year-old white man who underwent abdominal ultrasonography for lower urinary tract symp- toms evaluation. Computed tomography confirmed a $3.0 \mathrm{x}$ $2.8 \times 2.5 \mathrm{~cm}$ enhancing mass in the right retro-renal topography. Magnetic resonance imaging (MRI) showed a 3$\mathrm{cm}$ right retro-renal mass with high signal intensity on T2weighted images (Figure 3) and low signal intensity on T1weighted images. The patient underwent a mass excision through a right flank incision, and the right kidney was dissected free from the mass and spared. The postoperative period was uneventful. Macroscopic examination revealed a $3.0 \times 2.8 \times 2.5 \mathrm{~cm}$ capsulated, fibroelastic, bright-yellow mass. Microscopic examination, revealed focal areas of Antoni types A and B tissue. The cells were immunoreactive for $\mathrm{S} 100$ protein. The tumor was diagnosed as a schwannoma. At the 11-year follow-up, the patient remained asymptomatic, and there was no evidence of recurrence.

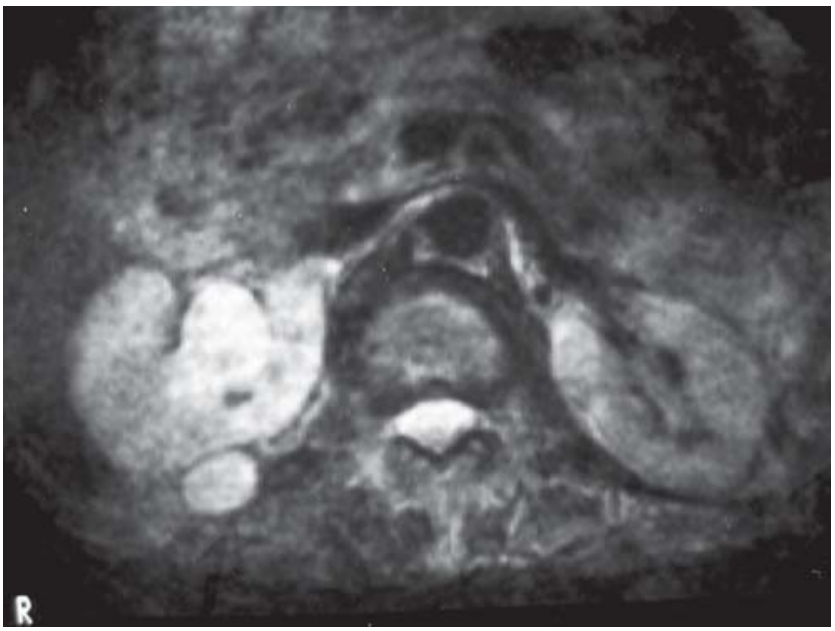

Figure 3 - T2-weighted abdominal MRI showing a 3 x 2.8 x 2.5-cm right retrorenal mass with high signal intensity.

\section{DISCUSSION}

Retroperitoneal schwannoma (also termed neurilemmomas or neurinomas) is a rare entity comprising only $0.5 \%$ to $12 \%$ of all retroperitoneal tumors. ${ }^{5}$ Most schwannomas are found in peripheral nerve fibers in the limbs, head, and neck. In the retroperitoneal position, they occur most commonly between 40 and 60 years of age, with a male/female ratio of 2:3. ${ }^{6}$ Diagnosis in the retroperitoneal position is difficult, and a large and deeply situated tumor is usually present before patients have any symptoms. The symptoms are vague and nonspecific, such as vague abdominal pain and dull ache. ${ }^{1,3}$ Atypical presentations are very rare and include flank pain and hematuria, headache, and secondary hypertension and recurrent renal colic pain. ${ }^{1}$ On gross appearance, schwannomas are usually solitary, well circumscribed, firm, smooth-surfaced tumors. Because of the larger size of extracranial schwannomas, these tumors are likely 
to manifest secondary to degenerative changes like cysts and calcification. Histologically, schwannomas consist of compact cellular lesions (Antoni type A tissue) and loose, hypocellular myxoid lesions with microcystic spaces (Anti type $\mathrm{B}$ tissue) ${ }^{6,7}$ The hallmark pattern of the benign variants is an alternation of these Antoni A and B areas, with a diffuse positivity for $\mathrm{S} 100$ protein in the cytoplasm of the tumor cells. ${ }^{1,3}$ Malignant degeneration of schwannomas is extremely rare, but when present, they act as high-grade sarcomas with a high likelihood of producing local recurrence and distant metastasis. The diagnosis of malignant peripheral nerve sheath tumor lacks standardized diagnostic criteria but features dense fascicules in a "marble-like" pattern consisting of asymmetrically tapered spindle cells. Malignancy is usually suggested histologically by mitosis, pleomorphism, and blood vessel infiltration. ${ }^{8}$

Radiological studies are fundamental in the diagnostic evaluation. Computed tomography scans typically show welldefined low or mixed attenuation with cystic necrotic central areas. Cystic changes occur more commonly in retroperitoneal schwannomas (up to 66\%) than in other retroperitoneal tumors. ${ }^{9}$ Other degenerative changes, such as calcification, hemorrhage, and hyalinization, can also be present. MRI findings of schwannomas have been reported as masses of low signal intensity on T1-weighted images and high signal intensity on T2-weighted images. ${ }^{6,10}$ These findings are characteristic but not specific of schwannomas and have been reported present in only $57 \%$ of the cases in previous studies, ${ }^{10}$ hindering the correct diagnosis. Additionally, the signal intensity on T2-weighted images may vary depending on cell density. Tumors with microscopic findings of hypercellular Antoni type A tissue have intermediate signals, while tumors with Antoni type B tissue have a bright signal on T2-weighted images. Additionally, other neurogenic tumors, such as ganglioneuroma, appear more commonly in the retroperitoneum than schwannomas and have similar findings on CT and MRI. ${ }^{11}$ Therefore, misdiagnosis of retroperitoneal schwannomas is not uncommon, and preoperative diagnosis as hepatic tumors, pancreatic cystic tumors, and psoas abscess have been reported. ${ }^{5,11,12}$

CT-guided core biopsy and fine needle aspiration have been founded to be unreliable for the diagnosis of retroperitoneal schwannoma. ${ }^{5,14}$ They may be helpful only if the sample contains enough Schwann cells to visualize microscopically. However, in areas of degeneration, the cellular pleomorphism can hinder the diagnosis, and degenerative cells may be misinterpreted as malignancy. One also runs the risk of hemorrhage, infection, and tumor seeding; thus, many authors do not recommend CT-guided biopsy. $3,7,15$ Therefore, surgical resection is the only accurate approach for pathologic evaluation to enable diagnosis of retroperitoneal schwannoma. Recent advances in laparoscopic instruments and skills have made laparoscopy an excellent approach for biopsy and even surgical resection of benign retroperitoneal tumors, aiding the diagnosis of these lesions.

Even though the best management of retroperitoneal neural sheath tumors is complete excision, considerable controversy exists over negative soft tissue margins. ${ }^{3,16}$ Some argue for complete surgical excision that may include, if necessary, the sacrifice of adjacent tissue and viscera. ${ }^{3,4,17}$ It is argued that the local recurrence rate ranges from $16 \%$ to $54 \%$ after conservative intralesional enucleation. ${ }^{18}$ Additionally, because malignancy cannot be excluded accurately preoperatively or even intraoperatively with frozen section analysis, complete surgical resection so as to attain negative margins is recommended. ${ }^{3,19}$ Others believe that because this is a benign mass, a simple enucleation or partial excision of the tumor is sufficient and have reported no increase in the size of schwannoma during a 6- and 14-year period. ${ }^{20,21}$ The argument here is that the morbidity associated with resection of adjacent tissue would not be justified in the treatment of a benign lesion. ${ }^{21}$ In our cases 2 and 3, the retroperitoneal masses were easily isolated from adjacent structures, and the lesions were completely ressected with negative margins. In case 1 , the mass was tightly adherent to the left kidney, and we preferred an en bloc resection because of the greater mass diameter and because malignancy could not be excluded.

Although the prognosis for retroperitoneal benign schwannoma is extremely good, malignant transformations have been reported, usually associated with von Recklinghausen 's disease. ${ }^{16}$ There are a few reported cases in which metastases occurred after resection of a histologically benign schwannoma. Therefore, it is suggested that careful monitoring is necessary after removal of benign retroperitoneal schwannomas. ${ }^{6}$

\section{REFERENCES}

1. Singh V; Kapoor R. Atypical presentation of benign retroperitoneal schwannoma: Report of three cases with review of literature. Int Urol and Nephrol. 2005;37:547-9.

2. Parfitt Jr HG, Hamonnd ME. Midletton Jr AW. Perirenal malignant schwannoma: a case report and review of literature. J Urol. 1982;128:1299-301.
3. Daneshmand S, Youssefzadeh D, Chamie K, Boswell W, Wu N, Stein JP, et al. Benign retroperitoneal schawannoma: A case series and review of literature. Urology. 2003;62:993-7.

4. Girgin C, Ozkan U, Sezer A, Tugyan N. A. Large pelvic schwannoma causing bilateral hydronephrosis. Int J Urol. 2003;10:616-8. 
5. Kishi Y, Kajiwara S, Seta S, Kawauchi N, Suzuki T, Sasaki K. Retroperitoneal schwannoma misdiagnosed as a psoas abscess: report of case. Surg Today. 2002;32:849-52.

6. Ohigashi T, Nonaka S, Nakanoma T, Ueno M, Deguchi N. Laparoscopic treatment of retroperitoneal benign schwannoma. Int J Urol. 1999:6;1003.

7. Enzinger FM; Weiss Sw. Benigm tumors of peripheral nerves. In: Enzinger FM, Weiss SW, editors. Soft tissue tumors. 2nd ed. St Louis: Mosby; 1988. p. 725-35

8. Chen KT; Latorrace R; Fubich D; Padgug A; Hafez G; Gilbert EF. Malignant schwannoma: A light microscopy and ultrastructural study. Cancer. 1980;45:1583-93.

9. Takatera H, Takiuchi H, Namiki M, Takaha M, Ohnishi S, Sonoda T. Retroperitoneal schwannoma. Urology. 1986;28:529-31.

10. Hayasaka K, Tanaka Y, Soeda S, Huppert P, Claussen CD. MR findings in primary retroperitoneal schwannoma. Acta radiol. 1999;40:78-82.

11. Radin R, David CL, Goldfarb H, Franncis IR. Adrenal and extra adrenal ganglioneuroma: Imaging findings in 13 adults. Radiology. $1997 ; 202: 703-7$

12. Nakamura M, Takayama T, Takayasu K, Shimada K, Yamamoto J, Kosuge T, et al. Retroperitoneal schwannoma mimicking hepatic tumor in the caudade lobe. Jpn J Clin Oncol. 1997;27:282-4.
13. Hsiao WC, Lin PW, Chang KC. Benign retroperitoneal schwannoma mimicking a pancreatic cystic tumor: case report and literature review. Hepato-Gastroenterology. 1998;45:2418-20.

14. Guz BY, Wood DP, Montie Je, Pontes JE. Retroperitoneal neural sheath tumors: Cleveland Clinic experience. J Urol. 1989;142:1434-7.

15. Neifer R and Nguyen GK. Aspiration cytology of solitary schwannoma Acta Cytol. 1985;29:12-14.

16. Ghosh BC, Ghosh L, Huvos AG, Fortner JG. Malignant schwannoma: a clinicopathologic study. Cancer. 1973;31:184-190.

17. Miller PL, Tessler A, Alexander S, Pinck BD. Retrtoperitoneal neurilemmoma. Urology. 1978;11:619-23.

18. Andonian S, Karakiewicz PI, Herr HW. Presacral cystic schwannoma in a man. Urology. 2003;62:8-10.

19. Giglio M, Giasotto V, Medica M, Germinale F, Durand F, Queirolo G, e al. Retroperitoneal ancient schwannoma: case report and analisys of clinico-radiological findings. Ann Urol. 2002;36:104-6.

20. Regan JF, Juler GL, Schmutzer KJ: Retroperitoneal neurilemoma. Am J Surg. 1977;134:140-5.

21. Gubbay AD, Moschilla G, Gray BN, Thompson I. Retroperitoneal schwannoma: a case series and review. Aust N J Surg. 1995;65:197200 